\title{
STDP-based Unsupervised Feature Learning using Convolution-over-time in Spiking Neural Networks for Energy-Efficient Neuromorphic Computing
}

\author{
GOPALAKRISHNAN SRINIVASAN, PRIYADARSHINI PANDA, and KAUSHIK ROY, \\ Purdue University, USA
}

\begin{abstract}
Brain-inspired learning models attempt to mimic the computations performed in the neurons and synapses constituting the human brain to achieve its efficiency in cognitive tasks. In this work, we propose Spike Timing Dependent Plasticity-based unsupervised feature learning using convolution-over-time in Spiking Neural Network (SNN). We use shared weight kernels that are convolved with the input patterns over time to encode representative input features, thereby improving the sparsity as well as the robustness of the learning model. We show that the Convolutional SNN self-learns several visual categories for object recognition with limited number of training patterns while yielding comparable classification accuracy relative to the fully connected SNN. Further, we quantify the energy benefits of the Convolutional SNN over fully connected SNN on neuromorphic hardware implementation.
\end{abstract}

CCS Concepts: • Computing methodologies $\rightarrow$ Neural networks; $•$ Hardware $\rightarrow$ Spintronics and magnetic technologies;

Additional Key Words and Phrases: Convolutional spiking neural network, convolution-over-time, stdp, unsupervised feature learning, energy-efficient neuromorphic computing

\section{ACM Reference format:}

Gopalakrishnan Srinivasan, Priyadarshini Panda, and Kaushik Roy. 2018. STDP-based Unsupervised Feature Learning using Convolution-over-time in Spiking Neural Networks for Energy-Efficient Neuromorphic Computing. F. Emerg. Technol. Comput. Syst. 14, 4, Article 44 (November 2018), 12 pages.

https://doi.org/10.1145/3266229

\section{INTRODUCTION}

Spiking Neural Networks (SNNs), widely regarded as the third generation of neural networks, offer a promising solution to approaching brain's processing capability for cognition. With more biologically realistic perspective on input processing, SNNs perform neural computation by means of spikes in an event-driven fashion. The asynchronous event- or spike-based computations make SNNs an energy-efficient choice over present-day artificial machine-learning models that operate with continuous inputs. Furthermore, SNNs, on account of spike-based processing, can be trained

This work was supported in part by the Center for Brain Inspired Computing (C-BRIC), one of the six centers in JUMP, a Semiconductor Research Corporation (SRC) program sponsored by DARPA, by the Semiconductor Research Corporation, the National Science Foundation, Intel Corporation, and the DoD Vannevar Bush Fellowship.

Authors' address: G. Srinivasan, P. Panda, and K. Roy, Purdue University, School of Electrical and Computer Engineering, 465 Northwestern Ave., West Lafayette, IN, 47907, USA; emails: \{srinivg, pandap, kaushik\}@purdue.edu.

Permission to make digital or hard copies of all or part of this work for personal or classroom use is granted without fee provided that copies are not made or distributed for profit or commercial advantage and that copies bear this notice and the full citation on the first page. Copyrights for components of this work owned by others than ACM must be honored. Abstracting with credit is permitted. To copy otherwise, or republish, to post on servers or to redistribute to lists, requires prior specific permission and/or a fee. Request permissions from permissions@acm.org.

(C) 2018 Association for Computing Machinery.

1550-4832/2018/11-ART44 \$15.00

https://doi.org/10.1145/3266229 
in an unsupervised manner using Spike Timing Dependent Plasticity (STDP) [1]. SNNs have attracted multi-dimensional research efforts ranging from algorithms to devices, circuits, and architectures in a quest to realize computationally efficient neuromorphic systems capable of real-time on-chip learning. At the algorithmic level, shallow fully connected SNN [5] and shallow/deep convolutional SNN [7, 10, 14, 16-19, 21, 27, 28, 30] have been proposed to achieve comparable accuracy with the state-of-the-art deep learning networks. At the hardware level, custom Complementary Metal-oxide Semiconductor (CMOS)-based architectures like SpiNNaker [13], IBM TrueNorth [20], and Intel Loihi [4] have been proposed to address the von-Neumann bottleneck inherent in the general-purpose computing platforms. However, CMOS-based implementations are area- and power-intensive due to the fundamental mismatch of the spiking neuronal and synaptic circuits with the underlying neuronal and synaptic dynamics. In this regard, emerging nanoelectronic devices capable of naturally mimicking the spiking neurons $[9,25,29]$ and synapses $[11,23,24,26]$ have been proposed as potential candidates for achieving much improved energy efficiency over CMOS-only realizations. In this work, we present STDP-based unsupervised feature learning using convolution-over-time in two-layer SNN and demonstrate its energy efficiency on neuromorphic hardware implementation.

Two-layer fully connected SNN [5], widely explored for unsupervised visual image recognition, essentially has all-to-all (full) synaptic connectivity between the input (or visual layer) and the output layer (or excitatory layer). STDP causes the synapses to self-learn the complete structure of the input patterns in the corresponding weights. The unsupervised learning paradigm presents an opportunity to create intelligent models that can learn with unlabeled data, making such SNN topologies attractive for on-chip implementations. However, the fully connected topology requires large number of neurons and synapses to self-learn varying representations of different classes of input patterns and attain competitive classification accuracy. This imposes the following limitation on crossbar-based neuromorphic hardware designs ideal for energy-efficient realization of SNNs. Large crossbars suffer from non-idealities arising from source and neuron resistances [3]. These issues become more prominent with fully connected SNNs that require large crossbar designs and hence constrain the scalability of a crossbar structure for complex recognition problems. Recent works on deep SNNs with hierarchical architecture, similar to the artificial deep learning networks, address the scalability issue. Deep SNNs presented in References [10, 16, 18, 21, 30] were trained using error backpropagation techniques that hinder on-chip learning. Researchers in References $[7,14,17]$ demonstrated STDP-based unsupervised training of the convolutional layers forming the deep SNN, which nevertheless requires an output layer trained with labeled data for inference.

We propose a two-layer Convolutional SNN that is capable of unsupervised feature learning and inference with sparser synaptic connectivity and limited number of training patterns. The proposed Convolutional SNN is composed of unique synaptic weight kernels connecting the input to every neuron in the excitatory layer. At any given time-step, a distinct segment of the input (image pattern) is modulated by the kernel weights to produce resultant current into the respective excitatory neurons. In the event of an excitatory neuronal spike, we perform STDP-driven updates on the interconnecting kernel weights that enables it to encode the corresponding input features. The updated synaptic weight kernels stride across the input over several time-steps and effectively incorporate input weight sharing to self-learn low-level features characterizing the input patterns. On account of the shared weight kernel learning with STDP, each excitatory neuron discovers general input representations by associating similarities across varied intra-class inputs to yield an improved and robust SNN with $4 \times$ lesser plastic synapses that require up to $7.5 \times$ fewer training patterns relative to the fully connected SNN (under iso-accuracy conditions) for the network sizes considered in this work. The decrease in the number of plastic synapses leads to feature learning with fewer excitatory neuronal spikes and synaptic weight updates, both of which enhance the 


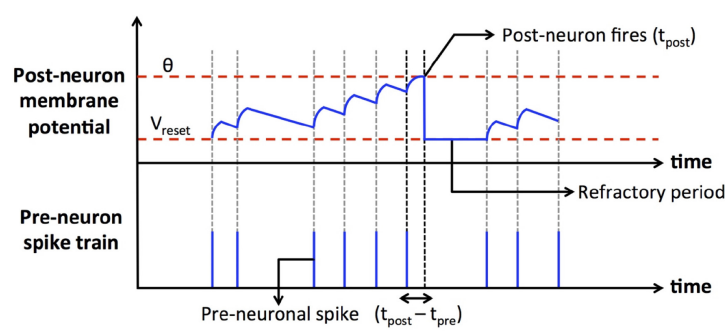

(a)

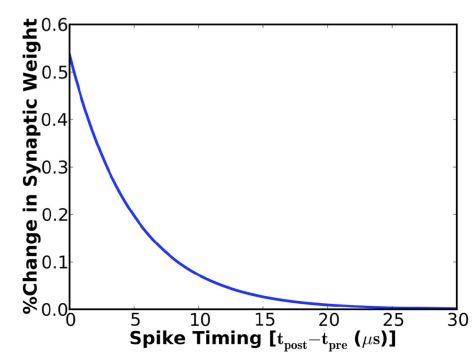

(b)

Fig. 1. (a) Leaky integrate-and-fire dynamics of a spiking neuron. At any given time-step, the input preneuronal spikes are modulated by the synaptic weights to generate resultant current into the post-neuron. This raises the post-neuronal membrane potential that subsequently leaks in the absence of any input spikes. The post-neuron fires an output spike (at time instant, $t_{\text {post }}$ ) when its membrane potential crosses a specific threshold $(\theta)$. The potential is thereafter reset (to $V_{\text {reset }}$ ) and the neuron is prevented from spiking for a certain refractory period. (b) Change in the synaptic weight based on the timing correlation between a pair of pre- and post-neuronal spikes $\left(t_{\text {post }}-t_{\text {pre }}\right)$ as mandated by the STDP model formulated in Equation (1), for learning rate $(\eta)$ of 0.01 , time constant $(\tau)$ of $5 \mu \mathrm{s}$, STDP offset $\left(S T D P_{\text {offset }}\right)$ of zero, maximum synaptic weight $\left(w_{\max }\right)$ of unity, current weight $(w)$ of 0.5 , and exponential factor $(\mu)$ of 0.9 .

computational efficiency in on-chip realizations. We demonstrate the improved energy efficiency offered by the Convolutional SNN on neuromorphic hardware implementation.

\section{BACKGROUND ON SPIKING NEURAL NETWORKS}

\subsection{Computational Model of Spiking Neuron and Synapse}

The fundamental building block of an SNN is a spiking (post) neuron connected by weighted synapses to a set of input (pre) neurons. The input pre-neuronal spikes are modulated by the synaptic weights to produce resultant post-synaptic current that leaks exponentially in the time interval between successive input spikes. The post-synaptic current is termed excitatory (inhibitory) postsynaptic current if the pre-neurons are excitatory (inhibitory). We use the Leaky Integrate-and-Fire (LIF) model described in Reference [5] and illustrated in Figure 1(a) to emulate the spiking neuronal dynamics. The post-neuron integrates the excitatory and inhibitory post-synaptic currents into its membrane potential, which decays exponentially following the removal of the input spikes. The neuron fires an output spike when its membrane potential exceeds a definite firing threshold. The potential is subsequently reset, the firing threshold is raised, and the neuron is restrained from spiking for a certain ensuing time interval designated as the refractory period. These mechanisms collectively regulate the neuronal spiking activity and prevent few neurons from entirely dominating the learning process, thereby facilitating competitive learning in SNNs.

\subsection{Synaptic Plasticity}

Spike Timing Dependent Plasticity (STDP) is popularly used to achieve unsupervised learning in SNNs. STDP postulates that the strength (or weight) of a synapse depends on the degree of timing correlation between the pre- and post-neuronal spikes. In this work, we use the power-law weight-dependent STDP model [5] illustrated in Figure 1(b) and described by

$$
\Delta w_{S T D P}=\eta \times\left(e^{-\frac{t_{\text {post }}-t_{\text {pre }}}{\tau}}-S T D P_{\text {offset }}\right) \times\left(w_{\max }-w\right)^{\mu},
$$

where $\Delta w_{S T D P}$ is the change in the synaptic weight, $\eta$ is the learning rate, $t_{\text {pre }}$ and $t_{\text {post }}$ are, respectively, the time instant of a pair of pre- and post-neuronal spikes, $\tau$ is the STDP time constant, 


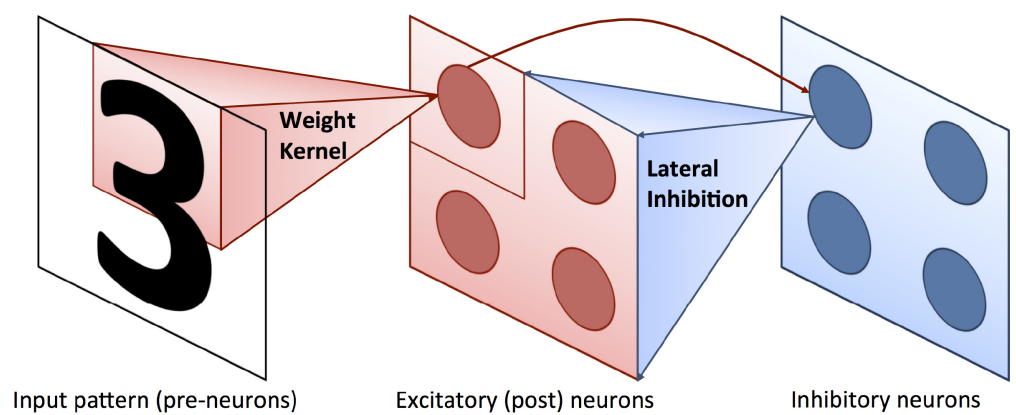

Fig. 2. Convolutional SNN topology for pattern recognition. The input neurons are connected via unique synaptic weight kernels to the excitatory neurons that further possess one-to-one synaptic connectivity with the inhibitory neurons. Each of these neurons inhibits all the excitatory neurons except the one from which it receives a forward synaptic connection.

$w_{\max }$ is the maximum constraint imposed on the synaptic weight, and $w$ is the current weight. The synaptic weight is increased (potentiated) if a pre-neuron triggers the connected post-neuron to fire within a period of time specified by the $S T D P_{\text {offset }}$, which signifies a strong causal relationship between the corresponding pair of neurons. The synaptic weight is decreased (depressed) for larger spike time differences. Further, the amount of weight update has an exponential dependence (governed by $\mu$ in Equation (1)) on the current weight. Larger the current weight, smaller is the ensuing weight change and vice versa. The non-linear weight updates ensure gradual change in the synaptic weight towards the maximum or minimum bound, which is desirable for efficient learning.

\section{PROPOSED CONVOLUTIONAL SNN TOPOLOGY AND LEARNING METHODOLOGY}

We propose Convolutional SNN composed of sparsely connected input and excitatory layers for efficient feature extraction and inference. We use weight kernels to achieve sparse synaptic connectivity between the input and every excitatory post-neuron as shown in Figure 2. It is worth mentioning that the dimension of the weight kernel is smaller than the resolution of the input pattern. We exploit the sharing of synaptic weights among the input neurons to extract salient features embedded in the input patterns using the STDP-based convolution-over-time learning methodology that will be described in the following sub-section. It is important to note that each excitatory neuron has a unique weight kernel that is trained to acquire attributes characterizing a specific class of input patterns. The excitatory neurons are further connected to the inhibitory neurons in a one-to-one manner. An excitatory neuronal spike eventually triggers the corresponding inhibitory neuron, which then inhibits the rest of the neurons in the excitatory layer. Lateral inhibition facilitates an excitatory neuron to spike exclusively for a particular class of input patterns, thereby enabling the synaptic weight kernel to self-learn distinctive input features.

\subsection{STDP-based Convolution-over-time Learning Methodology}

In this sub-section, we detail the proposed STDP-based convolution-over-time learning methodology for training the synaptic weight kernels. We begin by describing the learning methodology for a representative weight kernel connecting the input to a single excitatory neuron and subsequently demonstrate its capability for training various kernels in a network of such spiking neurons. Consider a randomly initialized weight kernel connecting a segment of the presented input pattern to an excitatory post-neuron as shown in Figure 3. Note that each image pixel constitutes an input 

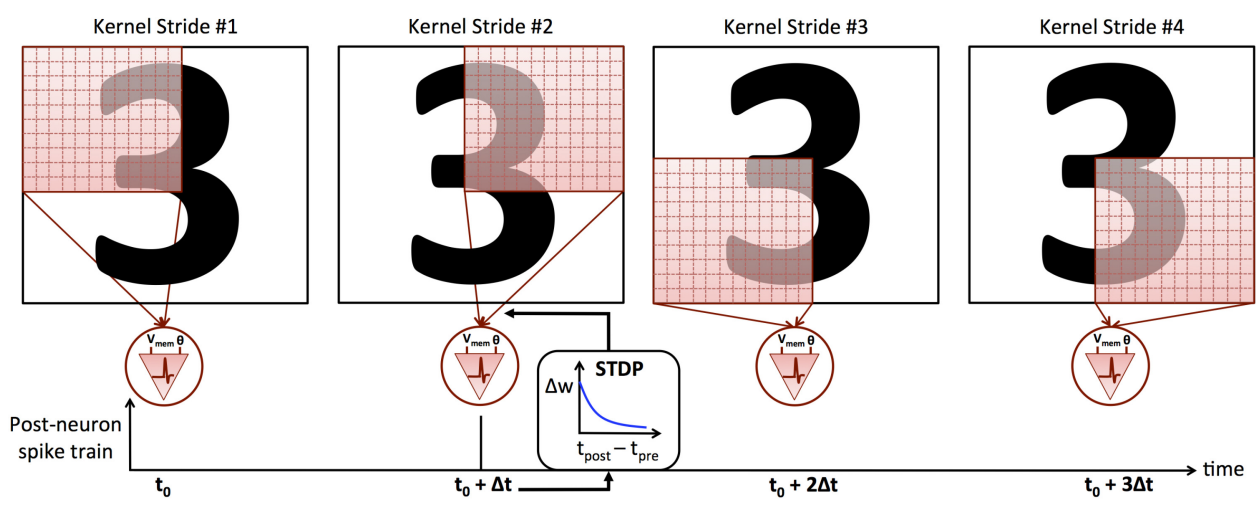

Fig. 3. Illustration of the STDP-based convolution-over-time learning methodology for training the synaptic weight kernel connecting the input to an excitatory post-neuron. At every time-step, the pre-neurons belonging to a distinct region in the input pattern are modulated by the kernel weights to generate resultant current into the post-neuron. This increases the neuronal membrane potential $\left(V_{m e m}\right)$, which triggers an output spike if the potential exceeds a definite threshold $(\theta)$. The kernel weights are modified at the instant of a post-neuronal spike ( $t_{0}+\Delta t$ in this example) based on the timing correlation with the corresponding pre-spikes as stipulated by STDP. The weight kernel is moved over (convolved with) the input pattern by pre-determined number of strides (four in this example) over multiple time-steps. This operation is repeated over the entire time duration for which the input pattern is presented.

pre-neuron whose firing rate is proportional to the corresponding pixel intensity. At any given time, the input pre-neuronal spikes are modulated by the kernel weights to produce resultant current into the post-neuron, which increases the neuronal membrane potential. The post-neuron fires an output spike only if its potential exceeds a definite threshold. In the absence of a postneuronal spike, the kernel is simply moved over the image by a pre-specified number of strides. However, in the event of a post-neuronal spike (at time instant $t_{0}+\Delta t$ in Figure 3), the kernel weights are modified based on the timing correlation between the post-spike and the corresponding pre-spikes as specified by the STDP rule formulated in Equation (1). The updated weight kernel influences the neuronal spiking dynamics at the following time instant. The weight kernel is subsequently moved over the entire image by a fixed number of horizontal and vertical strides over multiple time-steps. In other words, the weight kernel is convolved with the input pattern over several instants of time depending on the number of strides. The convolution operation is performed repeatedly until the end of the training duration. In the presented learning methodology, it is important to note that the weight kernel is connected to a distinct region in the input image at every time-step. Hence, updating the weight kernel at various instants of a post-neuronal spike enables it to encode features located in different image segments. The sharing of synaptic weights among the input neurons effectively causes the weight kernel to learn an overlapped representation of the characteristic features in the presented pattern.

Next, we demonstrate the applicability of the proposed learning mechanism for training the weight kernels associated with every excitatory neuron in a Convolutional SNN. At any instant of time, the various weight kernels are convolved with pre-neurons belonging to a specific region in the input image to activate the respective post-neurons. However, the SNN topology is equipped with lateral inhibitory connections that causes a distinct excitatory neuron to spike at a higher rate and consequently learn the presented input pattern. Lateral inhibition, in effect, differentiates the receptive field of every neuron in the excitatory layer and helps achieve competitive learning. At the end of the training phase, the weight kernel associated with each neuron learns salient features 


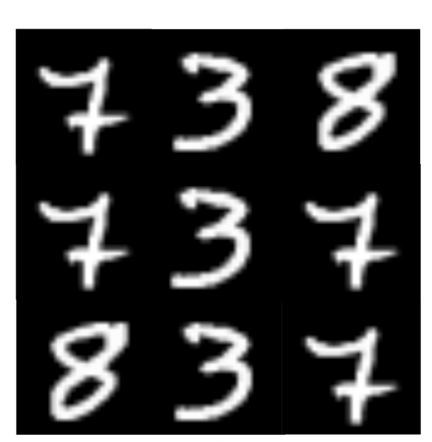

(a)

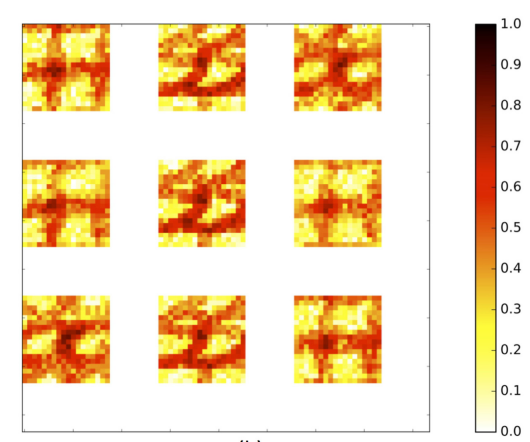

(b)

Fig. 4. (a) MNIST digit patterns (arranged in $3 \times 3$ grid) used to train a Convolutional SNN of 10 excitatory neurons. (b) Synaptic weight kernels ( $18 \times 18$ in size) associated with each of the 10 neurons (organized in $3 \times 3$ grid) trained using the STDP-based convolution-over-time learning methodology on the corresponding digit patterns.

embedded in a unique class of input patterns. This is illustrated in Figure 4, which shows the weight kernels learned by a Convolutional SNN of 10 excitatory neurons trained on a subcategory of digit patterns from the MNIST dataset [15]. We use the kernel representation of digit patterns " 3 ," " 7 ," and " 8 " to intuitively highlight the feature extraction capability of the presented learning methodology. It can be seen from Figure 4(b) that the kernel associated with " 3 " learned distinct semi-closed loops, while that linked to " 8 " acquired a couple of circular loops. The kernel related to "7" encoded the individual horizontal and vertical lines in the digit pattern. We show in the results section that the characteristic features thus learned can effectively be used to infer various input patterns in multi-class pattern recognition tasks.

\section{SIMULATION FRAMEWORK}

The Convolutional SNN was functionally simulated using BRIAN [8], an open-source Pythonbased SNN simulator, for different pattern recognition tasks. The leaky integrate-and-fire dynamics of the spiking neurons were modeled using differential equations described in Reference [5] with parameters adopted from Reference [12] except the time constant of the excitatory and inhibitory post-synaptic currents. We used a time constant of 1 and $10 \mu$ s for the excitatory and inhibitory post-synaptic currents during the training phase to increase the impact of lateral inhibition and achieve efficient learning. The time constants were, respectively, changed to 4 and $2 \mu$ s during the testing phase for increasing the excitatory neuronal spiking activity. Note that the simulation time step is $0.5 \mu \mathrm{s}$. The simulator was augmented to effect sparse connectivity between the input and excitatory neurons using synaptic weight kernels, each of which is convolved with an input pattern over multiple simulation time-steps. The kernel weights are modified at the instants of every spike fired by the connected post-neuron based on the timing correlation with the corresponding pre-spikes. The STDP-based convolution-over-time learning methodology causes the randomly initialized weight kernels to encode characteristic features pertaining to distinct classes of input patterns. At the end of the training phase, each excitatory neuron is tagged as having learned a particular input class for which it spiked the most during training. Each test pattern is predicted to belong to the class represented by the group of neurons with the highest average spike-count throughout the simulation period. The classification is accurate if the actual class of a test image matches with that predicted by the network of spiking neurons. The classification accuracy of the trained network is evaluated on the testing dataset. 
Table 1. Functional Simulation Parameters

\begin{tabular}{lccc}
\hline \hline Parameters & Convolutional SNN & Fully connected SNN & Convolutional SNN \\
\hline Dataset & MNIST & MNIST & Caltech \\
Simulation time-step & $0.5 \mu \mathrm{s}$ & $0.5 \mu \mathrm{s}$ & $0.5 \mu \mathrm{s}$ \\
Maximum Input Firing Rate & $63.75 \mathrm{spikes} / \mathrm{ms}$ & $63.75 \mathrm{spikes} / \mathrm{ms}$ & $204 \mathrm{spikes} / \mathrm{ms}$ \\
Simulation Time per Image & $350 \mu \mathrm{s}$ & $350 \mu \mathrm{s}$ & $350 \mu \mathrm{s}$ \\
STDP Learning Rate $(\eta)$ & 0.01 & 0.0025 & 0.01 \\
STDP Time Constant $(\tau)$ & $5 \mu \mathrm{s}$ & $10 \mu \mathrm{s}$ & $5 \mu \mathrm{s}$ \\
STDP offset $\left(S T D P_{\text {offset }}\right)$ & 0 & 0 & 0.0015 \\
Maximum weight $\left(w_{\max }\right)$ & 1 & 1 & 1 \\
Exponential factor $(\mu)$ & 0.9 & 0.9 & 0.1 \\
\hline \hline
\end{tabular}

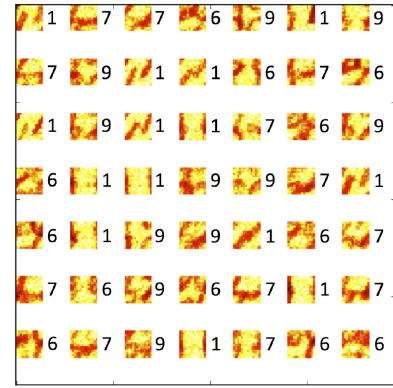

(a)

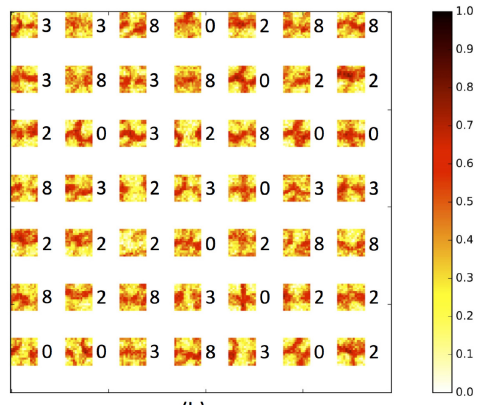

(b)

Fig. 5. (a) Weight kernels ( $14 \times 14$ in size) and the associated output classes (digits) learned by a network of 50 excitatory neurons (organized in $7 \times 7$ grid) trained on the subset \{ " 1 ," "6," "7," "9"\} using 40 examples from each category. (b) Weight kernels ( $14 \times 14$ in size) and the corresponding output classes learned by a similarly sized network trained on the subset \{“0," "2," "3," " 8 "\} using 40 examples from each category.

\section{RESULTS}

\subsection{Performance Analysis of Convolutional SNN on the MNIST Dataset}

The MNIST dataset [15] consists of 60,000 training and 10,000 testing handwritten digit patterns, each of which is $28 \times 28$ in dimension. We begin our analysis using a smaller network trained on a subset of output classes to illustrate the feature extraction capability of the Convolutional SNN. A smaller albeit sufficiently complex training subset is primarily chosen to be able to comprehensively analyze various kernel sizes and strides at a reasonable simulation speed for identifying the optimal configuration. We subsequently compare the classification accuracy of the optimized Convolutional SNN with that of a similarly sized fully connected SNN to show its efficacy in pattern recognition tasks. Finally, we train the Convolutional SNN on all the output classes for demonstrating its applicability in larger multi-class recognition tasks. The relevant simulations parameters are listed in Table 1.

In our first experiment, we trained a Convolutional SNN of 50 excitatory neurons using 40 unique patterns each of digits " 1, , "6," " 7 ," and " 9 ." The selected training subset consists of patterns with common features (" 1, , " 7, ," "9") along with those that are complementary (“9," "6"). It can be seen from Figure 5(a) that the weight kernel associated with each neuron encoded features corresponding to a specific output class (printed alongside each kernel). Our simulations further indicated that the impact of lateral inhibition needed to be high for efficient feature learning. We 


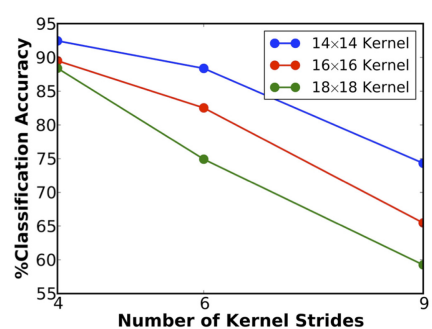

(a)

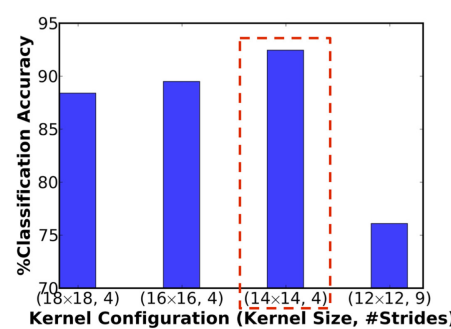

(b)

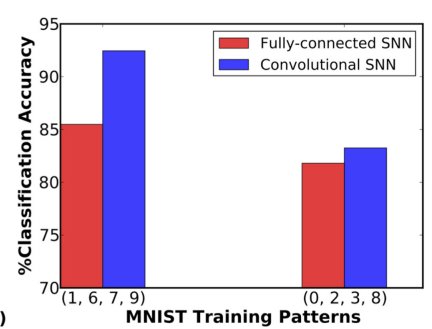

(c)

Fig. 6. (a) Classification accuracy of a Convolutional SNN of 50 excitatory neurons trained using 40 unique patterns each of digits " 1, ," 6 ," " 7 ," and "9" versus the number of kernel strides. (b) Classification accuracy of the network of 50 neurons for different kernel configurations including its size and the optimal number of strides. (c) Classification accuracy of the optimized Convolutional SNN topology (14× 14 kernel with 4 strides) and the baseline fully connected SNN individually trained on the subsets \{“1," "6," "7," "9" $\}$ and $\{$, 0 ," "2," "3," "8” $\}$ using 40 distinct examples from each class of digits.

note that a stronger inhibitory strength causes a distinct excitatory neuron to spike at a higher rate and predominantly learn a given input pattern while inhibiting the rest of the network. This enables the STDP-based learning mechanism to precisely adapt the weight kernel connecting each excitatory neuron. The trained network offered a classification accuracy of $92.45 \%$ on all the 4,000 images of " 1 ," "6," " 7 ," and "9" in the MNIST testing dataset, which illustrates the capability of the Convolutional SNN to distinguish between patterns with common features. A similar analysis on a network of 50 neurons using a relatively complex training subset consisting of the classes " 0 ," "2," "3," and " 8 " (Figure 5(b)) provided a classification accuracy of $83.25 \%$.

Next, we perform a rigorous analysis on a Convolutional SNN of 50 excitatory neurons using the training subset comprising digits " 1, , " 6 ," " 7 ," and " 9 " to ascertain the optimal kernel size and the number of horizontal $(h)$ and vertical $(v)$ strides required to cover the entire input pattern. We investigated strides of $4(2 h, 2 v), 6(3 h, 2 v)$, and $9(3 h, 3 v)$ for three different sizes of the synaptic kernel $(18 \times 18,16 \times 16$, and $14 \times 14)$. Our results (Figure 6(a)) indicate a drop in the classification accuracy as the number of strides is increased. The highest classification accuracy is obtained for a stride configuration of 4 for all the kernel sizes under investigation. In other words, we find that it is desirable to have minimal number of strides needed to cover the input pattern for a given size of the weight kernel. For instance, a $14 \times 14$ kernel requires a minimum of 4 strides while a $12 \times 12$ kernel necessitates 9 strides over the MNIST image ( $28 \times 28$ in dimension). We now explore kernels of varying sizes, each of which is convolved with the input patterns using the optimal number of strides. We note from Figure 6(b) that the classification accuracy improves as the kernel size is reduced from $18 \times 18$ to $14 \times 14$ while degrading significantly $(>15 \%)$ for sizes of $12 \times 12$ and lower. We surmise that the favorable configuration for a synaptic kernel including its size and the number of strides is the one that results in minimal kernel overlap across adjacent strides over an input pattern. We observed in our simulations that an increase in kernel overlap during the striding operation leads to excessive weight updates, which deteriorate the learned features. For MNIST digit recognition, $14 \times 14$ kernel with 4 strides over distinct non-overlapping image regions offers the best classification accuracy. It is worth mentioning that decreasing the kernel size below $12 \times 12$ failed to elicit sufficient number of spikes over the simulation interval, thereby proving detrimental to synaptic learning. The optimized Convolutional SNN topology trained using 40 unique patterns on the subsets \{“1," "6," "7," "9"\} and \{“0," "2," "3," "8”\}, respectively, provided 7\% and $1.45 \%$ increase in classification accuracy over a fully connected network of equivalent size as shown in Figure 6(c). The comparable accuracy is achieved with limited number of training 

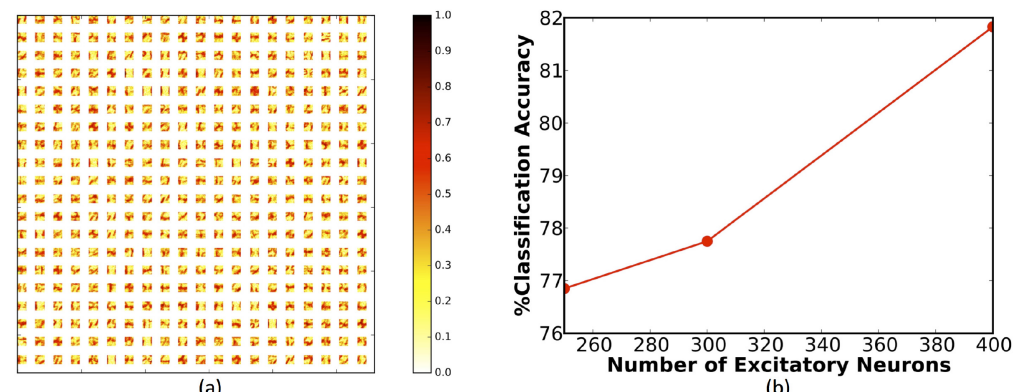

(b)

Fig. 7. (a) Weight kernels ( $14 \times 14$ in size) learned by a network of 400 excitatory neurons (organized in $20 \times 20$ grid) trained using 80 examples from each class of digit patterns from "0" to "9." (b) Classification accuracy of the optimized Convolutional SNN topology ( $14 \times 14$ kernel with 4 strides) trained on all the classes ("0"-"9") using 80 examples from each class against the number of excitatory neurons.

examples and $4 \times$ sparser synaptic connectivity between the input and excitatory neurons, where the sparsity metric is defined as the ratio of number of synaptic connections per neuron in a fully connected network to the kernel size in the Convolutional SNN topology.

Finally, we trained a bigger network on all the classes from " 0 " to " 9 " using the optimal kernel configuration previously determined $(14 \times 14$ kernel with $4(2 h, 2 v)$ strides) to validate its efficacy in larger multi-class recognition task. Figure 7(a) shows the distinct weight kernels learned by a network of 400 excitatory neurons trained with 80 examples from each class of digit patterns. We obtained a classification accuracy of $81.83 \%$ on the entire MNIST testing dataset while merely using 800 training examples out of a total of 60,000 . We note that a similarly sized fully connected SNN provided a classification accuracy of $80.98 \%$ for 800 training examples, which increased to $81.55 \%$ for 6,000 training examples. Thus, the ability of our Convolutional SNN to attain comparable classification accuracy using fewer training patterns for the investigated network sizes is a testament to its generalized feature learning capability rendered possible by the presented learning methodology. The classification accuracy can be improved further by increasing the number of excitatory neurons as evidenced by the trend in Figure 7(b).

\subsection{Performance Analysis of Convolutional SNN on the Caltech Dataset}

In an effort to further highlight the generalized feature learning capability of the Convolutional SNN, we train it to infer a subset of natural images from the Caltech dataset [6] consisting of 40 to 800 images each from 101 different object categories. We chose a subset containing a total of 2,080 images from 10 object categories, namely, yin yang, saxophone, stop sign, wrench, revolver, Buddha, airplane, pigeon, motorbike, and umbrella. We partitioned the subset into a training set containing 1,730 images and a testing set containing the remaining 350 images. The original Caltech images are stored in high resolution ( $300 \times 200$ in dimension) RGB colorspace. We preprocessed the Caltech images using the OpenCV library in Python [2] based on the methodology adopted in Reference [22] that is described below. We converted the Caltech images from RGB to grayscale format, averaged the pixels using a $3 \times 3$ Gaussian kernel for noise suppression, and downsized the images to a dimension of $28 \times 28$. We used 30 images from each object category to train a Convolutional SNN composed of 100 excitatory neurons and optimal kernel configuration ( $14 \times 14$ kernel with $4(2 h, 2 v)$ strides). The Convolutional SNN provided a classification accuracy of $80.29 \%$ on the testing set, which increased to $82 \%$ by uniformly scaling the firing threshold of the excitatory neurons by a factor of 1.03. A similarly sized fully connected SNN trained on all the 1730 images is reported to yield a classification accuracy of $84 \%$ [22], which is comparable to 


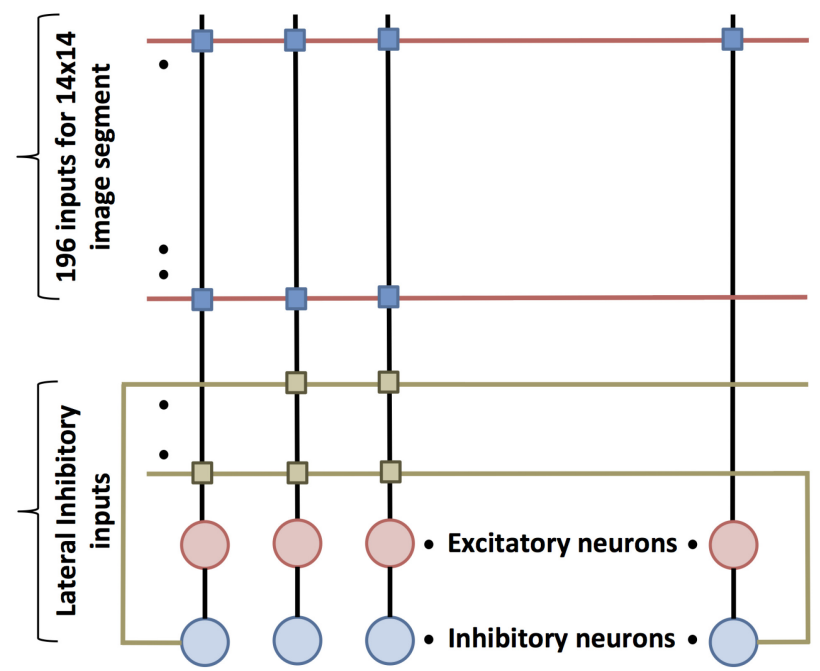

Fig. 8. Crossbar implementation of the Convolutional SNN with the input voltage-lines, driven by a segment of the input pattern $(14 \times 14$ in this sample illustration), connected to the excitatory neurons via weighted synapses. The excitatory neurons are directly connected to the corresponding inhibitory neurons that drive a negative voltage on the respective lateral inhibitory voltage-lines.

that provided by the Convolutional SNN considering the size of the testing set (350 images). It is important to note that our Convolutional SNN provided comparable classification accuracy while being trained on $\sim 5.8 \times$ fewer training examples, which is a testament to its generalized feature learning capability. Hence, the Convolutional SNN is a scalable topology for complex pattern recognition tasks with large number of classes.

\subsection{Energy Benefits of Convolutional SNN}

We evaluate the energy consumption of the Convolutional SNN on a crossbar implementation as illustrated in Figure 8. The input voltage-lines are driven by spike trains from a unique segment of the input pattern whose dimension depends on the size of the weight kernels. The input voltage spikes are modulated by the synaptic conductances to produce read-current into the respective excitatory neurons. An excitatory neuronal spike activates the corresponding column of synapses to update each individual conductance based on spike timing. Ag-Si memristive [11], phase-change [26], and spintronic devices $[23,24]$ have been demonstrated to efficiently realize the synaptic functionality in such crossbar architectures. The excitatory neurons are further connected to the inhibitory neurons in a one-to-one manner. An inhibitory neuronal spike drives a negative voltage on the corresponding row of inhibitory synapses. The synapses connecting the excitatory to inhibitory neurons and vice versa are not plastic and have unit connection weights.

Our system-level simulations demonstrated that the Convolutional SNN with $14 \times 14$ synaptic weight kernel connecting each excitatory neuron yields comparable classification accuracy relative to the fully connected SNN with $28 \times 28$ synapses feeding each excitatory neuron. The Convolutional SNN requires a total of 196 weight updates per excitatory neuronal spike as opposed to 784 weight updates necessitated by the fully connected SNN. Further, the Convolutional SNN produces an average of $\sim 6$ excitatory neuronal spikes per training pattern while the fully connected SNN yields $\sim 14$ excitatory neuronal spikes. The reduction in the excitatory neuronal spike-count offered by the Convolutional SNN can directly be attributed to the sparser synaptic connectivity 
between the input and excitatory neurons. We use the spintronic synapse proposed in Reference [24] to evaluate the energy consumption. Note that there could be potential degradation in the reported classification accuracies due to variation in the spintronic device conductance and other device imperfections like pinning of domain walls at defects, and so on. Reference [24] reported that write current of $\sim 80 \mu \mathrm{A}$ is needed for programming the spintronic synapse to its maximum conductance state. The maximum energy per synaptic weight update is $80 \mathrm{fJ}$ assuming that the write current is drawn from $1 \mathrm{~V}$ power supply and applied for a duration of $1 \mathrm{~ns}$. Consequently, the Convolutional SNN expends maximum write energy of $94.08 \mathrm{pJ}(80 \mathrm{fJ} \times 196$ kernel weights $\times 6$ excitatory neuronal spikes) per training pattern, which is $9.3 \times$ energy-efficient compared to the fully connected SNN that incurs $878.08 \mathrm{pJ}(80 \mathrm{fJ} \times 784$ synaptic weights $\times 14$ excitatory neuronal spikes) per training pattern. It is important to note that the energy benefit offered by the Convolutional SNN relative to the fully connected SNN is agnostic to the underlying hardware implementation, since it stems from the reduction in the number of trainable synapses and the excitatory neuronal spike-count per training pattern. Similar benefits in energy consumption could be expected in conventional CMOS implementations.

\section{CONCLUSION}

SNNs constitute a powerful computing paradigm as they offer a promising solution to approaching brain's efficient processing capability for cognition. Most unsupervised SNNs explored for pattern recognition use a fully connected topology that makes them inefficient with respect to synaptic storage. Moreover, the fully connected architecture fails to learn characteristic representations underlying the input patterns. As a result, they require training on a large fraction of input patterns that adds to the inefficiency of the learning model. In this work, we proposed Convolutional SNN composed of shared weight kernels that are convolved with the input patterns over time to learn representative input features in an unsupervised manner using STDP. The proposed Convolutional SNN offers lower storage requirement in addition to feature learning with limited number of training patterns. Our results on the MNIST and Caltech datasets indicated that Convolutional SNN consistently provides comparable accuracy relative to the fully connected SNN using $4 \times$ fewer synapses, resulting in possibility of up to $9.3 \times$ reduction in the energy consumption per training pattern compared to the fully connected SNN in neuromorphic hardware implementations.

\section{REFERENCES}

[1] Guo-qiang Bi and Mu-ming Poo. 1998. Synaptic modifications in cultured hippocampal neurons: Dependence on spike timing, synaptic strength, and postsynaptic cell type. F. Neurosci. 18, 24 (1998), 10464-10472.

[2] Gary Bradski. 2000. The opencv library. Dr. Dobb's f. Softw. Tools Prof. Programmer 25, 11 (2000), 120-123.

[3] Indranil Chakraborty, Deboleena Roy, and Kaushik Roy. 2017. Technology aware training in memristive neuromorphic systems based on non-ideal synaptic crossbars. arXiv Preprint arXiv:1711.08889.

[4] Mike Davies, Narayan Srinivasa, Tsung-Han Lin, Gautham Chinya, Yongqiang Cao, Sri Harsha Choday, Georgios Dimou, Prasad Joshi, Nabil Imam, Shweta Jain et al. 2018. Loihi: A neuromorphic manycore processor with on-chip learning. IEEE Micro 38, 1 (2018), 82-99.

[5] Peter U. Diehl and Matthew Cook. 2015. Unsupervised learning of digit recognition using spike-timing-dependent plasticity. Front. Comput. Neurosci. 9 (2015), 99.

[6] Li Fei-Fei, Rob Fergus, and Pietro Perona. 2007. Learning generative visual models from few training examples: An incremental bayesian approach tested on 101 object categories. Comput. Vision Image Understand. 106, 1 (2007), 59-70.

[7] Paul Ferré, Franck Mamalet, and Simon J. Thorpe. 2018. Unsupervised feature learning with winner-takes-all based STDP. Front. Comput. Neurosci. 12 (2018), 24.

[8] Dan Goodman and Romain Brette. 2008. Brian: A simulator for spiking neural networks in Python. Front. Neuroinfo. 2 (2008), 5. DOI : https://doi.org/10.3389/neuro.11.005.2008.

[9] Akhilesh Jaiswal, Sourjya Roy, Gopalakrishnan Srinivasan, and Kaushik Roy. 2017. Proposal for a leaky-integrate-fire spiking neuron based on magnetoelectric switching of ferromagnets. IEEE Trans. Electron. Dev. 64, 4 (2017), 1818-1824. 
[10] Yingyezhe Jin, Peng Li, and Wenrui Zhang. 2018. Hybrid macro/micro level backpropagation for training deep spiking neural networks. arXiv Preprint arXiv:1805.07866v1.

[11] Sung Hyun Jo, Ting Chang, Idongesit Ebong, Bhavitavya B. Bhadviya, Pinaki Mazumder, and Wei Lu. 2010. Nanoscale memristor device as synapse in neuromorphic systems. Nano Lett. 10, 4 (2010), 1297-1301.

[12] Florian Jug. 2012. On Competition and Learning in Cortical Structures. Ph.D. Dissertation. ETH Zurich.

[13] Muhammad Mukaram Khan, David R. Lester, Luis A. Plana, A. Rast, Xin Jin, Eustace Painkras, and Stephen B. Furber. 2008. SpiNNaker: Mapping neural networks onto a massively parallel chip multiprocessor. In Proceedings of the IEEE International Joint Conference on Neural Networks (IFCNN’08). Ieee, Hong Kong, China, 2849-2856.

[14] Saeed Reza Kheradpisheh, Mohammad Ganjtabesh, Simon J. Thorpe, and Timothe Masquelier. 2018. STDPbased spiking deep convolutional neural networks for object recognition. Neural Netw. 99 (2018), 56-67. DOI : https://doi.org/10.1016/j.neunet.2017.12.005

[15] Yann LeCun, Léon Bottou, Yoshua Bengio, and Patrick Haffner. 1998. Gradient-based learning applied to document recognition. Proc. IEEE 86, 11 (1998), 2278-2324.

[16] Chankyu Lee, Priyadarshini Panda, Gopalakrishnan Srinivasan, and Kaushik Roy. 2018. Training deep spiking convolutional neural networks with stdp-based unsupervised pre-training followed by supervised fine-tuning. Front. Neurosci. 12 (2018), 435.

[17] Chankyu Lee, Gopalakrishnan Srinivasan, Priyadarshini Panda, and Kaushik Roy. 2018. Deep spiking convolutional neural network trained with unsupervised spike timing dependent plasticity. IEEE Trans. Cogn. Dev. Syst. (2018), 1-1. DOI : https://doi.org/10.1109/TCDS.2018.2833071

[18] Jun Haeng Lee, Tobi Delbruck, and Michael Pfeiffer. 2016. Training deep spiking neural networks using backpropagation. Front. Neurosci. 10 (2016), 508.

[19] Timothée Masquelier and Simon J. Thorpe. 2007. Unsupervised learning of visual features through spike timing dependent plasticity. PLoS Comput. Biol. 3, 2 (2007), e31.

[20] Paul A. Merolla, John V. Arthur, Rodrigo Alvarez-Icaza, Andrew S. Cassidy, Jun Sawada, Filipp Akopyan, Bryan L. Jackson, Nabil Imam, Chen Guo, Yutaka Nakamura et al. 2014. A million spiking-neuron integrated circuit with a scalable communication network and interface. Science 345, 6197 (2014), 668-673.

[21] Priyadarshini Panda and Kaushik Roy. 2016. Unsupervised regenerative learning of hierarchical features in spiking deep networks for object recognition. In Proceedings of the International foint Conference on Neural Networks (IfCNN'16). IEEE, Vancouver, British Columbia, Canada, 299-306.

[22] Nitin Rathi, Priyadarshini Panda, and Kaushik Roy. 2018. STDP based pruning of connections and weight quantization in spiking neural networks for energy-efficient recognition. IEEE Trans. Comput.-Aided Des. Integr. Circ. Syst. (2018), 1-1. DOI : https://doi.org/10.1109/TCAD.2018.2819366

[23] Abhronil Sengupta, Zubair Al Azim, Xuanyao Fong, and Kaushik Roy. 2015. Spin-orbit torque induced spike-timing dependent plasticity. Appl. Phys. Lett. 106, 9 (2015), 093704.

[24] Abhronil Sengupta, Aparajita Banerjee, and Kaushik Roy. 2016. Hybrid spintronic-CMOS spiking neural network with on-chip learning: Devices, circuits, and systems. Phys. Rev. Appl. 6, 6 (2016), 064003.

[25] Abhronil Sengupta, Priyadarshini Panda, Parami Wijesinghe, Yusung Kim, and Kaushik Roy. 2016. Magnetic tunnel junction mimics stochastic cortical spiking neurons. Sci. Rep. 6 (2016), 30039.

[26] Manan Suri, Olivier Bichler, Damien Querlioz, Olga Cueto, Luca Perniola, Veronique Sousa, Dominique Vuillaume, Christian Gamrat, and Barbara DeSalvo. 2011. Phase change memory as synapse for ultra-dense neuromorphic systems: Application to complex visual pattern extraction. In Proceedings of the IEEE International Electron Devices Meeting (IEDM'11). IEEE, Washington, DC, 4-4.

[27] Amirhossein Tavanaei, Timothée Masquelier, and Anthony S. Maida. 2016. Acquisition of visual features through probabilistic spike-timing-dependent plasticity. In Proceedings of the International foint Conference on Neural Networks (IfCNN'16). IEEE, Vancouver, British Columbia, Canada, 307-314.

[28] Johannes C. Thiele, Olivier Bichler, and Antoine Dupret. 2018. Event-based, timescale invariant unsupervised online deep learning with STDP. Front. Comput. Neurosci. 12 (2018), 46. DOI : https://doi.org/10.3389/fncom.2018.00046

[29] Tomas Tuma, Angeliki Pantazi, Manuel Le Gallo, Abu Sebastian, and Evangelos Eleftheriou. 2016. Stochastic phasechange neurons. Nature Nanotechnol. 11, 8 (2016), 693-699.

[30] Yujie Wu, Lei Deng, Guoqi Li, Jun Zhu, and Luping Shi. 2018. Spatio-temporal backpropagation for training highperformance spiking neural networks. Front. Neurosci. 12 (2018), 331. DOI : https://doi.org/10.3389/fnins.2018.00331

Received December 2017; revised June 2018; accepted August 2018 